\title{
RHIC polarization decay in FY15 pp Run due to polarization profile development
}

\author{
H. Huang, P. Adams
}

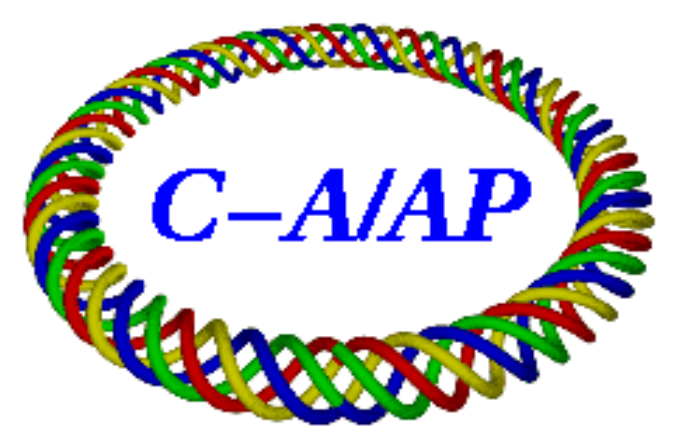

\section{Collider-Accelerator Department Brookhaven National Laboratory Upton, NY 11973}

\section{U.S. Department of Energy Office of Science, Office of Nuclear Physics}

Notice: This document has been authorized by employees of Brookhaven Science Associates, LLC under Contract No. DE-SC0012704 with the U.S. Department of Energy. The United States Government retains a nonexclusive, paid-up, irrevocable, world-wide license to publish or reproduce the published form of this document, or allow others to do so, for United States Government purposes. 


\title{
RHIC Polarization Decay in FY15 pp Run due to Polarization Profile Development
}

\author{
H. Huang, P. Adams \\ (Dated: May 23, 2016)
}

\begin{abstract}
The decay over time of ratio between polarization profile and beam profile has been analyzed in Ref.[1]. A follow up question is if we can get the decay of polarization profile and beam profile separately. With the beam profiles obtained from Ion Profile Monitor (IPM), this analysis was done and the results are analyzed. The results show that the contribution from polarization profile and beam profile is similar for yellow ring, but the contribution from polarization profile is much stronger in blue ring, which is consistent with lower polarization Blue ring.
\end{abstract}

\section{POLARIZATION PROFILE DEVELOPMENT}

The polarization profile and beam profile ratio $R$ is defined as [2]

$$
R=\left(\frac{\sigma_{b}}{\sigma_{p}}\right)^{2}
$$

where $\sigma_{b}$ is for the beam profile, and $\sigma_{p}$ is for the polarization profile. Gaussian beam distribution is assumed. For beam with depolarizing schemes in both $x$ and $y$ planes, there are two different $R$ values for the two planes. They can be different in general, but the observation is that both are about the same in AGS and RHIC. For round polarization profiles in both planes, the whole beam polarization $P_{\text {ave }}$ is given by

$$
P_{\text {ave }}=\frac{P_{0}}{(1+R)^{2}}
$$

where $P_{0}$ is the polarization with zero emittance. As the recent analysis[1] shows, the $R$ values are linearly going up against time in RHIC stores. This means that the polarization of the whole beam is going down with time (Eq. 2). As Eq.(1) shows, the whole beam polarization loss can come from two sources: either the transverse emittance increase or the polarization profile reduction. The second one is due to the high order depolarization resonances at store energy. One natural question is if the polarization profile dependence on time can be estimated from these data. Since emittance dependence on time can be derived from RHIC IPM measurement, the dependence of polarization profile on time can be derived. This analysis can provide quantitative information on the sources of polarization losses.

As a simple estimation, we assume round beam profile and round polarization profiles. Furthermore, the time dependence of these quantities are assumed as linear function. The $R$ values vs. time in store can be linearly fitted as function of time:

$$
R \approx R_{0}\left(1+a_{r} t\right) \approx\left[\frac{\sigma_{b 0}\left(1+a_{b} t\right)}{\sigma_{p 0}\left(1-a_{p} t\right)}\right]^{2}
$$

or

$$
\sqrt{R_{0}} \sqrt{\left(1+a_{r} t\right)} \approx \frac{\sigma_{b 0}\left(1+a_{b} t\right)}{\sigma_{p 0}\left(1-a_{p} t\right)} .
$$

In general, emittance will grow over time and polarization profile will be sharper over time. With above equation, both $a_{b}$ and $a_{p}$ will be positive numbers. The polarization profile dependence on time can be derived as

$$
\begin{array}{r}
\sigma_{p 0}\left(1-a_{p} t\right)=\frac{\sigma_{b 0}\left(1+a_{b} t\right)}{\sqrt{R_{0}} \sqrt{\left(1+a_{r} t\right)}} \\
\approx \frac{\sigma_{b 0}}{\sqrt{R_{0}}}\left[1+\left(a_{b}-\frac{a_{r}}{2}\right) t\right]
\end{array}
$$

The polarization profile increase (reduction of $\sigma_{p}$ ) factor $a_{p}$ is given by

$$
a_{p}=-\left(a_{b}-\frac{a_{r}}{2}\right)
$$




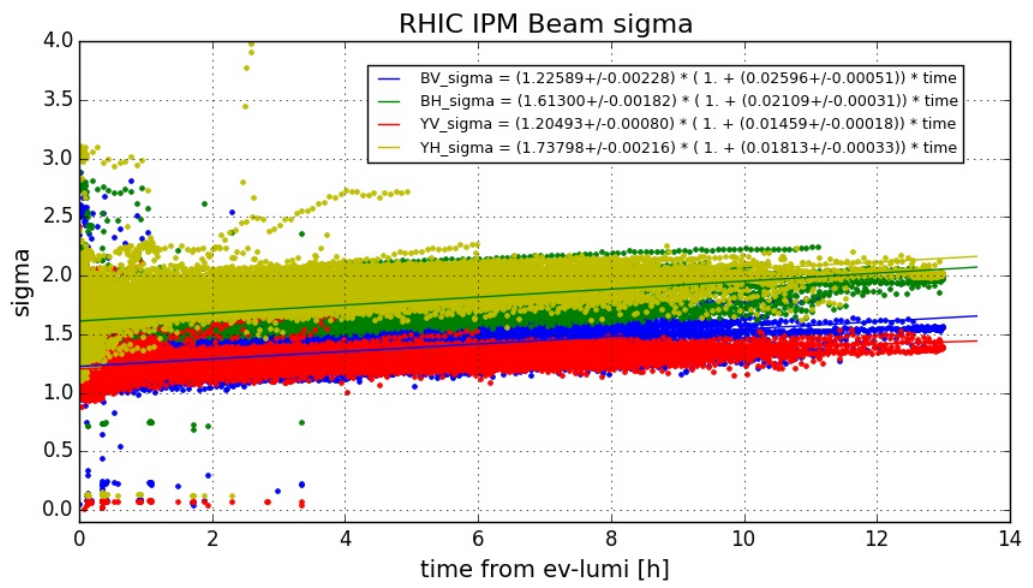

FIG. 1: The beam sigmas from RHIC IPM in both rings for all physics stores in run15 pp running period. The linear fits are for data points with store time up to 13 hours. A similar fit with data limited to store time less than 9 hours shows similar results, as the contribution from beyond 9 hours contributes very little.

\begin{tabular}{|c|ccccc|}
\hline & $R_{0}$ & $a_{r}$ & $\sigma_{b}$ & $a_{b}$ & $a_{p}$ \\
\hline Blue H & $0.0621 \pm 0.011$ & $0.216 \pm 0.056$ & $1.613 \pm 0.002$ & $0.0211 \pm 0.0003$ & \\
\hline Blue V & $0.0772 \pm 0.011$ & $0.161 \pm 0.037$ & $1.226 \pm 0.002$ & $0.0260 \pm 0.0005$ & \\
\hline Ave. Blue & $0.0700 \pm 0.008$ & $\mathbf{0 . 1 7 8} \pm \mathbf{0 . 0 3 1}$ & $1.462 \pm 0.001$ & $\mathbf{0 . 0 2 2 4} \pm \mathbf{0 . 0 0 3}$ & $\mathbf{0 . 0 6 6 6} \pm \mathbf{0 . 0 1 6 0}$ \\
\hline Yellow H & $0.0752 \pm 0.0018$ & $0.064 \pm 0.006$ & $1.738 \pm 0.002$ & $0.0181 \pm 0.0003$ & \\
\hline Yellow V & $0.0659 \pm 0.0166$ & $0.085 \pm 0.057$ & $1.205 \pm 0.001$ & $0.0146 \pm 0.0002$ & \\
\hline Ave. Yellow & $0.0751 \pm 0.0018$ & $\mathbf{0 . 0 6 4} \pm \mathbf{0 . 0 0 6}$ & $1.270 \pm 0.001$ & $\mathbf{0 . 0 1 5 4} \pm \mathbf{0 . 0 0 0 2}$ & $\mathbf{0 . 0 1 6 6} \pm \mathbf{0 . 0 0 3 0}$ \\
\hline
\end{tabular}

TABLE I: Averaged decay parameters for blue and yellow rings. They are highlighted as bold cases. Other parameters are listed for reference.

\section{RESULTS AND ANALYSIS}

The beam sizes at IPM locations are different from the ones at polarimeters due to the different beta functions. But the relative time dependence slope $\left(a_{b}\right)$ should be the same. We can use the slopes derived at IPM locations for the analysis of beam profiles at polarimeter locations. The beam sizes from IPM in both rings are plotted in Fig.1. Although the beam sizes may be different at the polarimeter locations, the relative slope of the beam size change should be the same. The initial beam sizes are similar in both rings for both planes. For the same ring, the slopes of both planes are similar, too, which justifies to average the two slopes for the analysis. It should be noted that only slopes are used in this analysis, not the initial values of the beam sizes. With the averaged slopes for beam profiles derived from IPM data, the slopes of polarization profiles are derived. They are listed in Table I. The $R_{0}$ is listed to show that indeed they are similar in $\mathrm{x}$ and $\mathrm{y}$ directions. The $\sigma_{b}$ values are listed to show that they are consistent in both rings. It does not matter what the true $\sigma_{b}$ value is, only the slope $a_{b}$ matters. For yellow ring, the contributions from beam emittance growth $\left(a_{b}\right)$ and polarization profile decay $\left(a_{p}\right)$ are similar. For blue ring, the contribution from $a_{p}$ is three times of that from $a_{b}$, which indicates stronger effect from higher order depolarizing resonance effect in blue. It should be noted that only $a_{b}$ and $a_{p}$ are used to derived the polarization decay slope $a_{p}$.

\section{SUMMARY}

Ideally, we would like to have quadratic term for R's time dependence. The data quality probably only can give us first order fit, unfortunately. The polarization loss has two sources: emittance growth, and the polarization loss due to (high order) depolarizing resonances. The contributions from the two sources are quantified from this analysis. As the results shows, more polarization loss in blue is correlated to much stronger effect from depolarizing resonances. In addition, the blue polarization decay problem is two folds: somewhat faster emittance growth but mainly stronger effect from depolarizing resonances at stores. It is worthwhile to consider polarization simulation study to compare with these results. 


\section{Acknowledgment}

The authors would like to thank W. Fischer for suggestions to carry out this study. The authors would also like to thank O. Eyser, A. Poblaguev, B. Schmidke and G. Webb for offline polarization data.

[1] H. Huang, P. Adams, C-A/AP/558, (BNL, 2016).

[2] W. Fischer and A. Bazilevsky, Phys. Rev. STAB 15. 041001(2012). 